\title{
STAT3-induced IncRNA HAGLROS overexpression contributes to the malignant progression of gastric cancer cells via mTOR signal-mediated inhibition of autophagy
}

\author{
Jin-Fei Chen ${ }^{1+}$, Peng $\mathrm{Wu}^{1+}{ }^{1 \dagger}$, Rui Xia ${ }^{2}$, Jian Yang ${ }^{1}$, Xin-Ying Huo ${ }^{1}$, Dong-Ying Gu' ${ }^{1}$ Cui-Ju Tang ${ }^{1}$, Wei De ${ }^{3}$
} and Fen Yang ${ }^{3^{*}}$ (B)

\begin{abstract}
Background: Long noncoding RNAs (IncRNAs) are an important class of functional regulators involved in human cancers development, including gastric cancer (GC). Studying aberrantly expressed IncRNAs may provide us with new insights into the occurrence and development of gastric cancer by acting as oncogenes or tumor suppressors. In this study, we aim to examine the expression pattern of IncRNA HAGLROS in GC and its clinical significance as well as its biological role in tumor progression.

Methods: Bioinformatics analysis and qRT-PCR were performed to detect the relative expression of HAGLROS in GC tissues and cell lines. Gain or loss of function approaches were used to investigate the biological functions of HAGLROS. The effect of HAGLROS on proliferation was evaluated by MTT, colony formation assay and nude mouse xenograft model. Wound healing and Transwell assays were used to study the invasion and migration of GC cells. FISH, RIP, RNAseq, Luciferase report assays, RNA pulldown and Western blot were fulfilled to measure molecular mechanisms. Results are shown as means \pm S.D. and differences were tested for significance using Student's t-test (two-tailed).
\end{abstract}

Results: We screened out HAGLROS, whose expression was significantly increased and correlated with outcomes of GC patients by publicly available IncRNAs expression profiling and integrating analyses. Exogenous down-regulation of HAGLROS expression significantly suppressed the cell proliferation, invasion and migration. Mechanistic investigations showed that HAGLROS was a direct target of transcriptional factor STAT3. Moreover, HAGLROS knockdown decreased mTOR expression and increased autophagy-related genes ATG9A and ATG9B expression. Further investigation showed that HAGLROS regulated mTOR signals in two manners. In the one hand, HAGLROS competitively sponged miR-100-5p to increase mTOR expression by antagonizing miR-100-5p-mediated mTOR mRNA inhibition. On the other hand, HAGLROS interacted with mTORC1 components to activate mTORC1 signaling pathway which was known to be an important negative signal of autophagy. Here activation of MTORC1 signaling pathway by HAGLROS inhibited autophagy, thereby promoted excessive proliferation and maintained the malignant phenotype of GC cells.

Conclusion: The present study demonstrates that HAGLROS overexpression contributes to GC development and poor prognosis and will be a target for GC therapy and further develop as a potential prognostic biomarker.

Keywords: IncRNA, HAGLROS, miR-100-5p, mTOR, Gastric cancer, Autophagy

\footnotetext{
* Correspondence: yangfen@njmu.edu.cn

${ }^{\dagger}$ Equal contributors

${ }^{3}$ Department of Biochemistry and Molecular Biology, School of Basic Medical

Sciences, Nanjing Medical University, Nanjing 211166, People's Republic of

China

Full list of author information is available at the end of the article
} 


\section{Background}

Recent evidence indicates that although more than $70 \%$ of the eukaryotic genome is transcribed, only approximately 1 to $2 \%$ of the transcriptome contributes to protein-coding RNA, suggesting that transcription is not limited to the protein-coding portion of the eukaryotic genome but includes other non-protein-coding sections $[1,2]$. Based on their sizes, these transcribed noncoding RNAs (ncRNAs) can be classified as small, medium and long. Small ncRNAs range from 18 to $31 \mathrm{bp}$ in length, whereas long ncRNAs range in size from $200 \mathrm{bp}$ to over several hundred kilobases. Medium ncRNAs measure between $31 \mathrm{bp}$ and $200 \mathrm{bp}$ and contain mainly snRNAs and snoRNAs. Long noncoding RNAs (lncRNAs) are of interest because emerging evidence indicates that a subset of long noncoding RNAs mediate their biological functions using chromatin as a substrate to interact with the genetic information encoded in the genome [3].

Gastric cancer (GC) is ranked the fifth most common malignant neoplasm in the world, with approximately 951,600 new diagnoses and 723,100 deaths in 2012 [4]. Despite the decreased mortality rate of GC in recent years, it is still the second most common cause of cancer death [5, 6]. Further exploration of the molecular mechanisms underlying GC occurrence and development is urgently needed. Studying the aberrantly expressed lncRNAs involved in signaling pathways in GC may provide us with new insights into the occurrence and development of this disease. By acting as oncogenes or tumor suppressors, lncRNAs contribute to GC occurrence and development. Several lncRNAs, such as HULC, MALAT1, IncRNA-ATB and HOTTIP, have been demonstrated oncogenic activity [7-11], while other lncRNAs, including HOTAIR, GAS5 and PTENP1, are considered tumor suppressors [12-14]. The cellular localization of lncRNAs is varied. Majority of lncRNAs are localized to the nucleus (MALAT1 and NEAT1), some are distinctively found in the cytoplasm (DANCR and OIP5-AS1), and certain lncRNAs are found in both locations (TUG1, CasC7 and HOTAIR) [15]. The cellular localization of lncRNAs is intended for indulging in range of physiological activities from chromatin remodeling to translational regulation [16]. The basic structural and interactive capabilities of IncRNAs with other cellular biomolecules can help distinguish and specifically reveal their central roles in tumorigenesis. LncRNA-DNA, IncRNA-RNA and IncRNA-protein interactions are especially important. IncRNAs are involved in various levels of regulation, including transcriptional repression by binding to the PRC2 (Polycomb Repressive Complex 2) [12]. LncRNAs can also serve as a 'sponge' to titrate miRNAs, thus participating in posttranscriptional processing $[17,18]$. The most important biomolecular interactions of lncRNAs are with RNAbinding proteins. All the classic molecular mechanisms of lncRNAs, such as guiding, scaffolding and decoying, are ultimately executed through interactions with proteins $[19,20]$.

The mammalian target of rapamycin (mTOR) signaling pathway integrates both intracellular and extracellular signals and functions as a central regulator of cell metabolism, growth, proliferation and survival. Activation of the mTOR pathway has a substantial regulatory role in cell proliferation and cell cycle progression [21]. The mTOR protein forms at least two distinct multiprotein structures: mTOR complex 1 (mTORC1) and 2 (mTORC2). These complexes share the catalytic mTOR subunit, mammalian lethal with sec-13 protein 8 (mLST8, also known as GbL), the DEP domain containing mTOR-interacting protein (DEPTOR), and the Tti1/Tel2 complex. Regulatoryassociated protein of mammalian target of rapamycin (raptor) and proline-rich Akt substrate $40 \mathrm{kDa}$ (PRAS40) are specific to the mTORC1 complex, whereas rapamycininsensitive companion of mTOR (rictor), mammalian stress-activated map kinase-interacting protein 1 (mSin 1$)$ and protein observed with rictor 1 and 2 (protor $1 / 2$ ) are only part of the mTORC2 complex [21].

Here, we report the characterization of HAGLROS as an lncRNA highly expressed in GC and implicated in the regulation of cell proliferation and migration. In the present study, we identified HAGLROS by analyzing publicly available lncRNA expression profiling data from GC. HAGLROS has only one transcript, a $699 \mathrm{bp}$ lncRNA, according the NCBI (NCBI Reference Sequence: NR_110457.1). HAGLROS was up-regulated in $\mathrm{GC}$ tissues and served as an independent predictor for overall survival. In addition, HAGLROS was a direct transcriptional target of STAT3, and HAGLROS regulated GC cell proliferation both in vitro and in vivo. Mechanistic studies showed that HAGLROS regulated mTOR signaling by functioning as a competing endogenous RNA (ceRNA), which suppressed the degradation of mTOR mRNA by competing with miR100-5p. HAGLROS also functioned as an mTORC1 binding partner, interacting with mTOR, Raptor and PRAS40 and stabilizing the complex. Activated mTOR promoted excessive proliferation and maintained the malignant phenotype of GC cells by inhibiting autophagy. Taken together, our study revealed that STAT3-induced lncRNA HAGLROS overexpression contributes to the malignant proliferation and invasion of GC cells via mTOR signal-mediated inhibition of autophagy and predicts poor outcomes in GC patients. These results provide important experimental evidence for the diagnosis and treatment of GC and suggest that HAGLROS may serve as a target for new therapies in human GC. 


\section{Methods}

\section{Microarray analysis}

GC gene expression data were downloaded from The Cancer Genome Atlas (TCGA) and National Center for Biotechnology Information (NCBI) Gene Expression Omnibus (GEO) dataset. Heat maps representing differentially regulated genes were generated using Cluster 3.0 software (http://hemi.biocuckoo.org). Microarray data have been deposited under accession number GSE58828.

\section{Cell lines}

The human gastric cancer cell lines SGC-7901, BGC-823, HGC-27, MGC-803 and AGS and the normal gastric epithelium cell line (GES1) were obtained from the Chinese Academy of Sciences Committee on Type Culture Collection Cell Bank (Shanghai, China). They are cultivated in Dulbecco's modified Eagle's medium (DMEM) supplemented with $10 \%$ fetal bovine serum (FBS) in a humidified atmosphere containing $5 \% \mathrm{CO}_{2}$ at $37^{\circ} \mathrm{C}$.

\section{Study subjects}

We obtained 84 paired GC and adjacent non-cancerous tissues from patients who underwent surgery at Nanjing First Hospital of Jiangsu Province in China between 2011 and 2012 and who were diagnosed with GC based on histopathologic evaluation. No local or systemic treatment was conducted in these patients before surgery. All collected tissue samples were preserved in RNA Transport (OMEGA Engineering Inc., Norwalk, CT, USA) and immediately frozen at $-80{ }^{\circ} \mathrm{C}$ until required. The clinical characteristics of all patients are listed in Additional file 1: Table S1.

\section{Antibodies and reagents}

The following antibodies were used in the study: mTOR Pathway Antibody Sampler Kit, anti-P70S6K, anti-pP70S6K, anti-LC3, anti-P62 and secondary antibodies were purchased from Cell Signaling Technology (Danvers, MA, USA). 3-MA was purchased from Sigma-Aldrich (St. Louis, MO, USA).

\section{qRT-PCR analysis}

qRT-PCR was used to detect expression levels of HAGLROS and other genes in GC tissues and cells following the manufacturer's instructions (LightCycler ${ }^{\circ} 480$, Roche, Basel, Switzerland). GAPDH and $\beta$-actin were used as controls. Essential details referred to the MIQE guidelines [22]. Primers are listed in Additional file 2: Table S2. Total RNA was extracted using Trizol (Invitrogen, Grand Island, NY, USA), according the manufacturer's instructions. cDNA was synthesized using PrimeScript ${ }^{\mathrm{Th}} \mathrm{RT}$ reagent Kit (Takara Bio USA, Inc., Mountain View, CA, USA, No. RR047A).
For miRNA quantification, the Bulge-loop ${ }^{\mathrm{Tm}}$ miRNA qRT-PCR Primer Sets (one RT primer and a pair of qRT-PCR primers for each set) specific for miR-100-5p is designed by RiboBio (Guangzhou, China). cDNA was synthesized using PrimeScript ${ }^{\mathrm{Tm}}$ RT reagent Kit (Takara Bio USA, No. RR037A).

\section{Plasmid construction and cell transfection}

The full-length complementary cDNA of human HAGLROS was synthesized by Invitrogen and cloned into the expression vector pc-DNA3.1 (Takara Bio USA, Inc.) the small hairpin RNA (shRNA) of the HAGLROS was provided by Invitrogen Corporation (Grand Island, NY, USA), and the final construct was verified by sequencing. Plasmid vectors for transfection were prepared using DNA Midiprep Kits (Qiagen, Hilden, Germany) and transfected into GC cells using Lipofectamine 2000 (Invitrogen). The siRNAs were transfected into GC cells using Lipofectamine 2000 according the manufacturer's instructions. All siRNA and shRNA sequences are listed in Additional file 2: Table S2.

\section{Luciferase reporter assays}

The STAT3-binding motif in the promoter region of HAGLROS was identified by JASPAR (http://jaspar.genereg.net/). The different fragment sequences were synthesized and then inserted into the pGL3-basic vector (OMEGA Engineering Inc.) and co-transfected with STAT3 plasmid into $293 \mathrm{~T}$ cells. The miR-100-5p sequence was synthesized, inserted into the pGL3-basic vector and co-transfected with wild-type and mutant HAGLROS (the binding site for miR-100-5p was mutated) plasmid into $293 \mathrm{~T}$ cells. The 3 '-UTR of mTOR was cloned into the luciferase vector and transfected into 293T together with miR-100-5p mimics, the miR100-5p inhibitor, the HAGLROS plasmid or the negative control. All vectors were verified by sequencing, and luciferase activities were assessed using a Dual Luciferase Assay Kit (OMEGA Engineering Inc.) in accordance with the manufacturer's instructions.

\section{Cell proliferation}

Cell proliferation ability was examined using a Cell Proliferation Reagent Kit I (MTT, Sigma-Aldrich). Absorbance values were measured at the wavelength of $490 \mathrm{~nm}$. Inhibitory rates were calculated by Microsoft Excel (Microsoft Corporation, Redmond, WA, USA).

Colony formation assays were performed to monitor the cloning capability of GC cells. Cells were seeded in 6-well plates at $1 \times 10^{3}$ cells/well and cultivated in DMEM (without any cytokine) with $10 \%$ FBS for 14 days, with the medium being replaced every 4 days. Colonies were fixed with methanol and stained with $0.1 \%$ crystal violet (Sigma-Aldrich) in PBS for $15 \mathrm{~min}$. Colony 
formation was determined by counting the number of stained colonies.

\section{Cell migration and invasion}

A wound healing assay was used to test for cell migration capabilities. A total of $2-4 \times 10^{5}$ cells were seeded in 6-well plates, cultured for 12-24 h, and transfected with siRNAs or a control siRNA and with pc-DNA3.1HAGLROS or a control vector. Once cultures reached $85 \%$ confluency, the cell layer was scratched with a sterile plastic tip, washed with culture medium, and then cultured for $24 \mathrm{~h}$ and $48 \mathrm{~h}$. At different time points, images of the plates were acquired using a microscope (Olympus, Tokyo, Japan) and relative areas of wounds using Image J software to quantify and calculate the significance of the observed event.

For the invasion assays, $1 \times 10^{5}$ cells in serum-free medium were placed into the upper chamber of an insert coated with Matrigel. Medium containing 10\% FBS was added to the lower chamber. After incubation for $24 \mathrm{~h}$, the cells remaining on the upper membrane were removed with cotton wool. Cells that had migrated or invaded through the membrane were fixed with methanol, stained with $0.1 \%$ crystal violet, imaged, and counted using an inverted microscope (Olympus).

\section{Establishment of xenografts and in vivo studies}

Animal studies were performed in accordance with the criteria outlined in the 'Guide for the Care and Use of Laboratory Animals' prepared by the National Academy of Sciences and published by the National Institutes of Health (USA). Four-week-old female athymic BALB/c nude mice were maintained under specific pathogen-free conditions and manipulated according protocols approved by the Shanghai Medical Experimental Animal Care Commission and the Committee on the Ethics of Animal Experiments of the Nanjing Medical University. HAGLROS shRNA and Ctrl shRNA stably transfected SGC-7901 cells were harvested, and $1 \times 10^{7}$ cells were subcutaneously injected into a single side of each mouse. Tumor sizes were measured by caliper and recorded every 3 days. The tumor volumes were calculated from the length (the longest diameter across the tumor) and width (the corresponding perpendicular diameter) using the following formula: $\pi / 6 \times$ length $\times$ width $^{2}$. After 20 days of growth, animals were killed, and tumors were resected and preserved at $-80{ }^{\circ} \mathrm{C}$ or in formaldehyde for qRT-PCR and IHC staining, respectively.

\section{FISH and subcellular fractionation}

FISH assay was performed using a Ribo ${ }^{\text {Tn }}$ Fluorescent In Situ Hybridization Kit and Ribo ${ }^{\text {Tx }}$ lncRNA FISH Probe Mix (Ribo, Guangzhou, China) according to the manufacturer's protocols. The separation of nuclear and cytosolic fractions was performed using a PARIS Kit (Life Technologies, Carlsbad, CA, USA) according to the manufacturer's instructions.

\section{RNA immunoprecipitation}

An EZMagna RNA immunoprecipitation (RIP) Kit (Millipore, Bedford, MA, USA) was used following the manufacturer's protocol. BGC-823 and SGC-7901 cells were lysed in complete RIP lysis buffer (containing proteinase inhibitor and phosphatase inhibitor), and the cell extract was incubated with magnetic beads conjugated with specific antibodies or control IgG for $6 \mathrm{~h}$ at $4{ }^{\circ} \mathrm{C}$. Beads were washed and incubated with proteinase $\mathrm{K}$ to remove proteins. Finally, purified RNA was subjected to qRT-PCR analysis.

\section{RNA pull-down assay}

RNAs were in vitro transcribed using T7 RNA polymerase (Ambion Inc., Austin, TX, USA), purified using an RNeasy Plus Mini Kit (Qiagen), and treated with RNasefree DNase I (Qiagen). Transcribed RNAs were biotin labeled with Biotin RNA Labeling Mix (Ambion Inc.). Positive, negative, and biotinylated RNAs were mixed and incubated with BGC-823 cell lysates. Magnetic beads were added to each binding reaction, followed by incubation at room temperature. Beads were washed with washing buffer, and eluted proteins were examined by Coomassie brilliant blue (Beyotime, Shanghai, China) staining and Western blot analysis.

\section{Chromatin immunoprecipitation}

Chromatin immunoprecipitation (ChIP) experiments were performed using the MagnaChIP Kit (Millipore) according to the manufacturer's instructions as described previously [23]. ChIP assay related primers are listed in Additional file 2: Table S2.

\section{Western blot analysis and immunoprecipitation}

Western blot analysis and immunoprecipitation were performed according to standard protocols as described previously [24].

\section{LC3-II punctuation assay}

For detecting the LC3-II punctuation, BGC-823 cells were transiently co-transfected with GFP-LC3 and HAGLROS siRNAs for $24 \mathrm{~h}$ and then seeded in a 24well plate covered with $14 \times 14 \mathrm{~mm}$ slips for next $24 \mathrm{~h}$. After that, cells were fixed, permeabilized, and incubated with DAPI for 10 mins, and then adhered to coverslips after PBS washing. Cells on coverslips were observed using a confocal microscope (Olympus). 
Immunohistochemical (IHC) analysis

To quantify Ki67 expression, both the intensity and extent of immunoreactivity were evaluated and scored. IHC staining and score evaluation were performed according to standard protocols as described previously [25].

\section{Statistical analysis}

Differences between groups were assessed by a paired, two tailed Student's t-test. The Chi-square test was used to analyze the pathologic features of HAGLROS expression in GC. The survival curves are drawn using KaplanMeier survival plots and tested using log-rank tests. Univariate and multivariate Cox proportional hazards modeling was used to determine the effects of variables on survival. All statistical analyses were performed using SPSS 22.0 software (IBM SPSS, Chicago, IL, USA).

\section{Results}

Expression of HAGLROS is up-regulated in human GC tissues and correlates with poor prognosis

From analysis of microarray profile GSE58828 of GEO dataset and mapsoft (http://ncrnamap.mbc.nctu.edu.tw/ php/search.php), we identified differentially expressed 398 lncRNAs, including upregulated 82 lncRNAs (Fig. 1a). We selected the most differentially expressed 10 lncRNAs and validated their expression in 12 cases GC patient tissues. Results showed that HAGLROS is the most upregulated and well repeatedly expressed lncRNA (Additional file 3: Figure S1). Furthermore, we measured HAGLROS expression levels in tissue samples from 84 GC patients. qRT-PCR assays showed that HAGLROS expression was significantly higher in the cancer tissues than in the adjacent normal tissues (Fig. 1b). By analyzing clinic-pathological factors, we found that the high HAGLROS expression was correlated with poor prognosis in GC patients. There was an obvious positive correlation between higher HAGLROS levels and increased invasion depth and TNM stage (Fig. 1c and d). We divided samples into high (above the mean, $n=44$ ) and low (below the mean, $n=40)$ HAGLROS expression groups for analysis based on the median value of HAGLROS levels. Clinic-pathological factors between the two groups are shown in Additional file 1: Table S1. HAGLROS levels were also correlated with tumor invasion depth and TNM stage. No relation was found between HAGLROS expression and other factors, e.g., sex, age or histological grade. As shown in Fig. 1e, elevated HAGLROS levels predicted a poor prognosis in patients with GC. Multivariate analysis further revealed that HAGLROS expression could be regarded as a potential diagnostic biomarker for overall survival in patients with GC $(P=0.005)$, for TNM stage $(P=0.019)$ and for lymph node metastasis $(P=0.001)$ (Additional file 4: Table S3).
Elevated HAGLROS expression is associated with GC cell proliferation and invasion

HAGLROS expression was significantly higher in GC cell lines, including SGC-7901, BGC-823, HGC-27, MGC-803 and AGS, than in the normal gastric epithelium cell line GES-1 (Fig. 2a). To determine the function of increased HAGLROS expression in GC, we studied the effects of HAGLROS knockdown and overexpression on GC cell lines. The results showed that HAGLROS knockdown by siRNAs inhibited cell viability in BGC823 and SGC-7901 cells, which have higher HAGLROS expression. In contrast, HAGLROS overexpression by cDNA transfection increased cell viability in AGS cells, which have lower HAGLROS expression (Fig. 2b). HAGLROS knockdown decreased clone formation in BGC-823 and SGC-7901 cells, while HAGLROS overexpression promoted clone formation in AGS cells (Fig. 2c). In the wound scratch assay, HAGLROS knockdown decreased wound healing in both BGC-823 and SGC-7901 cells, and HAGLROS overexpression increased wound healing in AGS cells (Fig. 2d and Additional file 5: Figure S2a). HAGLROS knockdown decreased cell invasion in both BGC-823 and SGC-7901 cells, while HAGLROS overexpression strengthened invasion in AGS cells (Fig. 2e). Altogether, these findings suggested that increased HAGLROS expression in GC contributed to GC cell proliferation and migration. Transcription efficiencies of knockdown and overexpression were shown in the Additional file 5: Figure S2b.

\section{HAGLROS promotes GC cell tumorigenesis in vivo}

In a nude mice xenograft model, SGC7901 cells with recombinant adenoviral vector producing shRNA against HAGLROS were inoculated into the flanks of mice, and SGC7901 cells infected with adenoviral vector carrying control shRNA were inoculated into the opposite flank of the same mouse as a control. Ad-shRNAs significantly inhibited tumorigenesis in vivo, as tumor weight and size were obviously decreased compared with the controls (Fig. 3a-c). We considered shRNAs to be appropriate for HAGLROS knockdown until the subcutaneous tumors were harvested and tested for relative HAGLROS expression levels (Fig. 3d). Furthermore, we detected stronger Ki-67 expression in tumors derived from control shRNA expression than those derived from HAGLROS shRNA expression, and HE staining showed similar changes (Fig. 3e).

\section{STAT3 induces HAGLROS expression by functioning as a transcription factor}

The promoter region of HAGLROS was identified by the public website (http://www.ensembl.org/index.html) and we focused on the first 2000 bp upstream the transcription start site. We performed a computational 


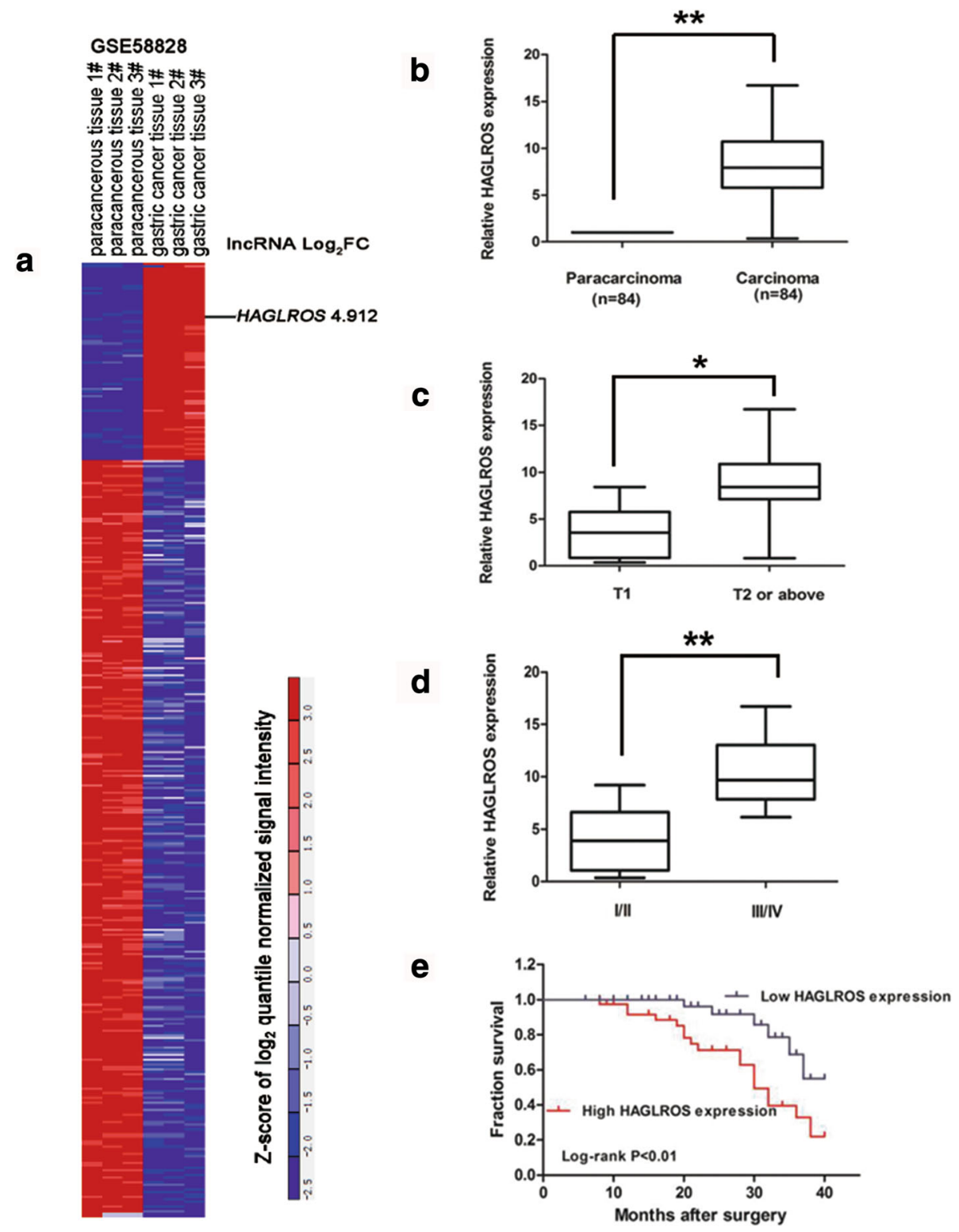

Fig. 1 InCRNA HAGLROS is overexpressed in GC tissues and associated with the outcome of GC patients. a Hierarchical clustering analysis shows InCRNAs that were differentially expressed (fold change $>2 ; P<0.05$ ) in GC and normal tissues from GEO datasets. $\mathbf{b}$ HAGLROS expression was analyzed by qRT-PCR in GC and adjacent non-tumor tissues $(n=84)$. The results are presented as the fold-change in tumor tissues relative to normal tissues. $\mathbf{c}$ and $\mathbf{d}$ Higher HAGLROS levels were positively correlated with advanced invasion depth and TNM stage. e Survival times of patients with high HAGLROS expression were decreased compared to those patients with low HAGLROS expression. ${ }^{*} P<0.05,{ }^{*} P<0.01$

screen based on computer algorithms (Jaspar: http://jaspar.genereg.net/) and found that 125 putative sites with scores $>10$ were predicted in the 2000 bp upstream of HAGLROS transcript. We measured 10 common transcriptional factors (TCF3, SP1, STAT3, SP3, KLF5, EN1, CDX1, STAT4, TCF12 and HOXB2) to detect HAGLROS expression upon knockdowns of potential transcriptional factors and found that only STAT3 had an obvious corresponding change. The putative E1 and E2 STAT3 binding sites located between -1874 to
-1864 bp (TTGCAGGGAAA) and between -418 bp to -408 bp (TTTTTAGGAAT) of the HAGLROS promoter, respectively (Fig. 4a). To verify whether high expression of HAGLROS was mediated by STAT3, we overexpressed STAT3 by cDNA transfection and knocked down STAT3 using an siRNA targeting STAT3. Upon STAT3 down-regulation, HAGLROS expression was markedly decreased in BGC-823 and SGC-7901 cells (Fig. 4b), while upon STAT3 overexpression, HAGLROS expression was significantly increased in 

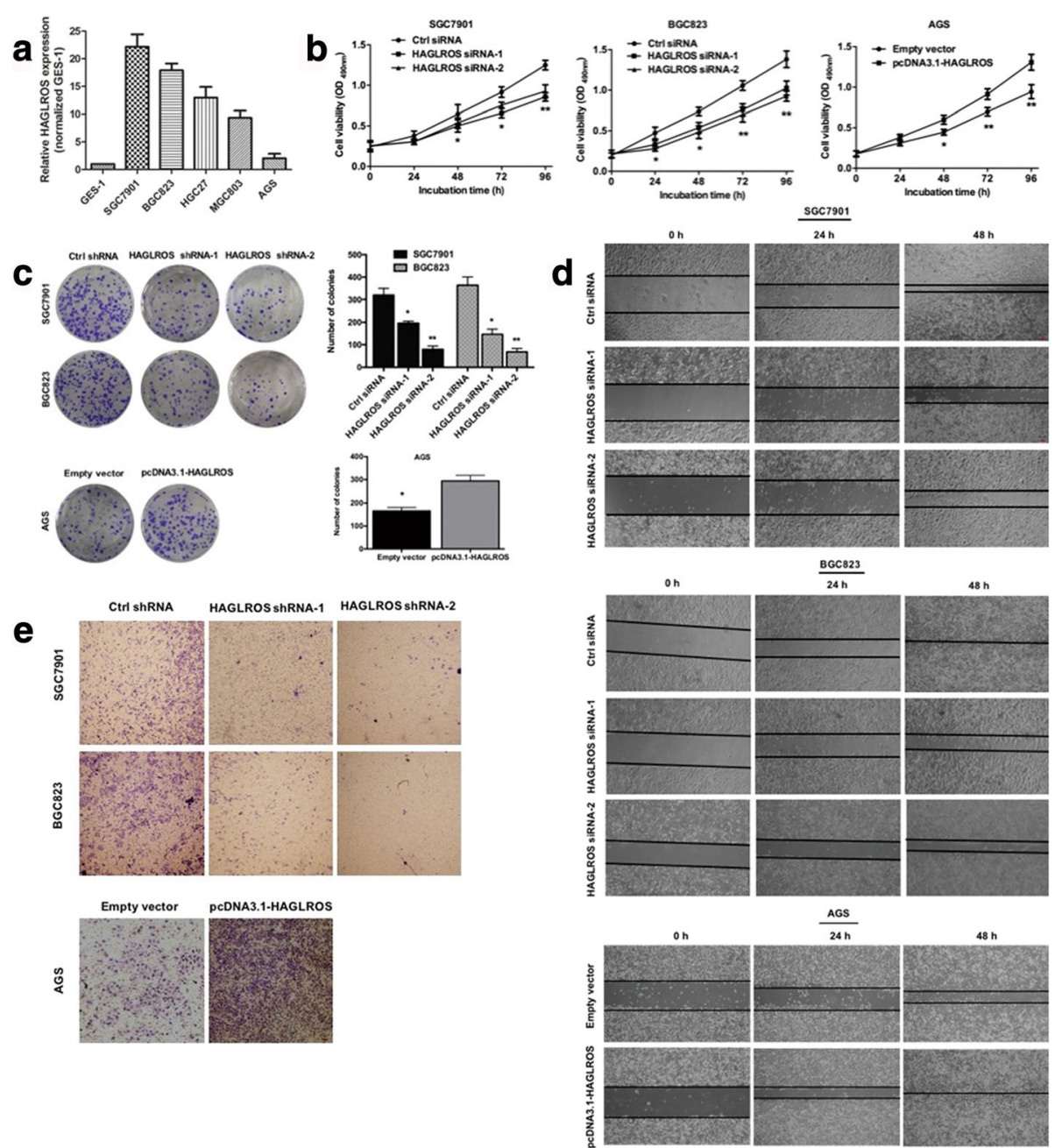

Fig. 2 HAGLROS regulates GC cell proliferation and invasion in vitro. a Analysis of HAGLROS expression levels in GC cell lines compared with GES1 cells by qRT-PCR. $\mathbf{b}$ Cell proliferation was determined by MTT assay after SGC-7901 and BGC-823 cells were transfected with siRNAs against HAGLROS and AGS cells were transfected with HAGLROS plasmid. c The representative results of colony formation assays using SGC-7901 and BGC-823 cells transfected with siRNAs against HAGLROS and AGS cells transfected with HAGLROS plasmid. $\mathbf{d}$ Cell migration was monitored by wound scratch assay; cell lines were treated the same as in (b) and (c). e Cell invasion was measured by Transwell assay; cell lines were treated the same as in (b) and (c)

BGC-823 and SGC-7901 cells (Fig. 4c). Chromatin immunoprecipitation (ChIP) assay was used to validate whether STAT3 could bind to the predicted site on the HAGLROS promoter region. The results showed that HAGLROS enrichment over input markedly increased STAT3 antibody levels compared to IgG antibody levels in BGC-823 and SGC-7901 cells (Fig. 4d and e).

To investigate whether STAT3 directly transcriptionally regulates HAGLROS expression, we cloned the full promoter region of HAGLROS and E1 or E2 promoter region mutants into luciferase reporter plasmids (Fig. 4f). As shown in Fig. 4g, luciferase assays showed that STAT3 induced the promoter activity of HAGLROS in 293T cells transfected with the full promoter region of HAGLROS. After transfection of the STAT3 expression plasmid, the
P1 mutant (not containing the E2 STAT3 binding site) caused a significant reduction in promoter activity compared to the full-length promoter construct, but this reduction was not seen in the P2 mutant (not containing the E1 STAT3 binding site). These results indicate that the STAT3 binding site in the promoter region of HAGLROS is located the $\mathrm{E} 2$ binding site ( $-418 \mathrm{bp}$ to $-408 \mathrm{bp})$ instead of the E1 binding site ( -1874 bp to $-1864 \mathrm{bp})$.

\section{HAGLROS, as a mainly cytoplasmic IncRNA, serves as a sponge for miR-100-5p}

To investigate the mechanism by which HAGLROS contributed to the malignant phenotypes of GC cells, we studied the localization of HAGLROS based on its activity within different subcellular areas. After GC cells were 


\section{a}
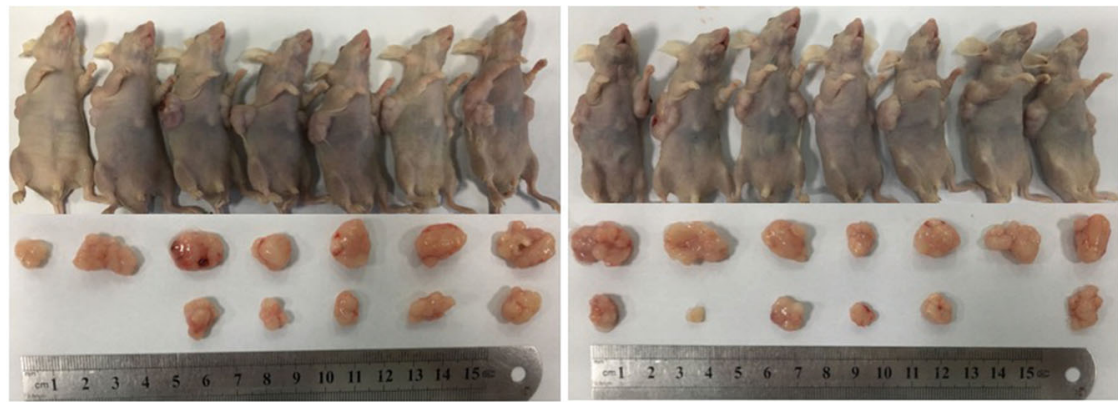

b
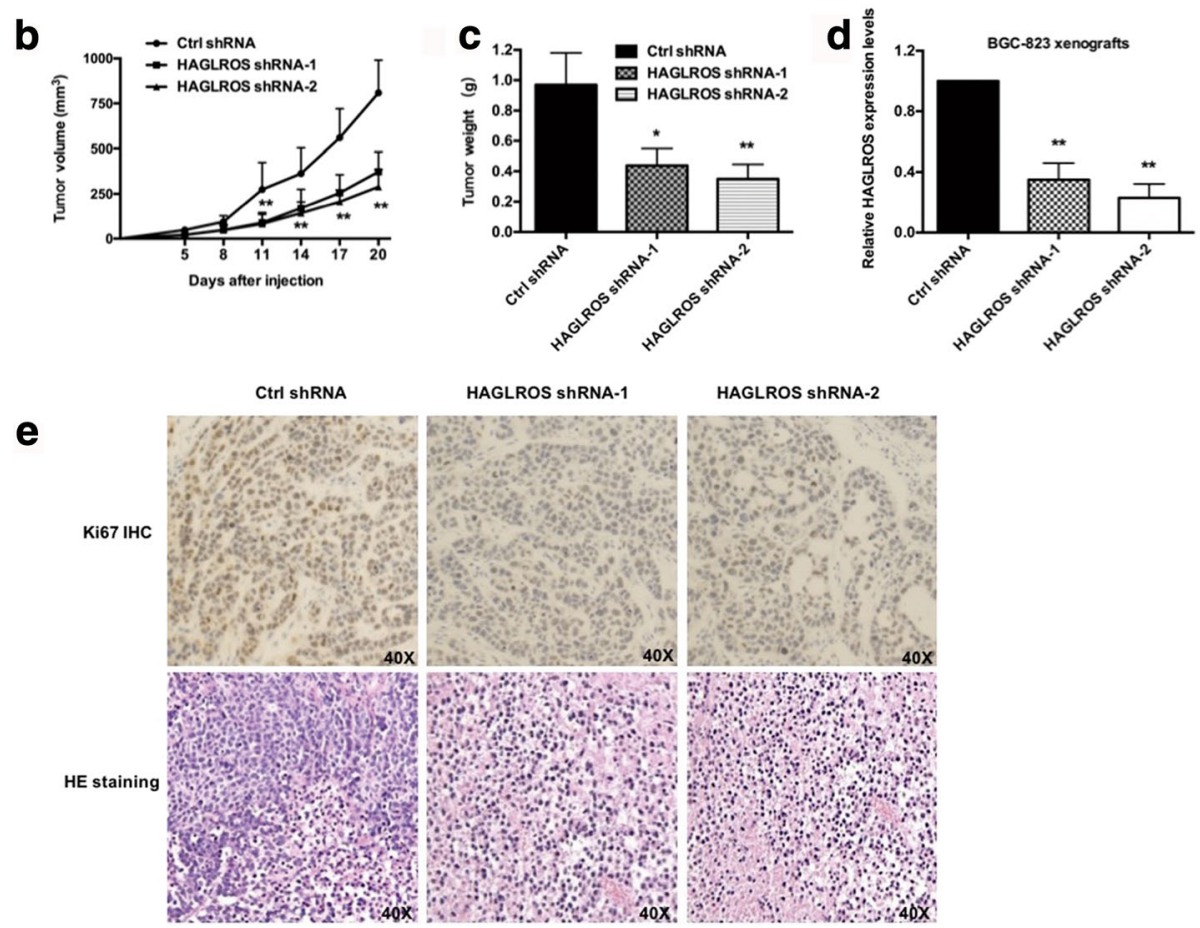

Fig. 3 HAGLROS promotes GC cell tumorigenesis in vivo. a BGC-823 cells transfected with Ctrl shRNA and HAGLROS shRNA were injected respectively into nude mice $(n=7)$, which were killed by carbon dioxide euthanasia 20 days after injection. b Tumor volumes were calculated every 3 days beginning 5 days after injection. Bars indicate SD. c Tumor weights were represented as the means of tumor weights \pm SD. $\mathbf{d}$ Transfection efficiency was tested by qRT-PCR. e The tumor sections underwent IHC staining using antibodies against Ki-67 and HE staining. Error bars indicate means \pm S.E.M. ${ }^{*} P<0.05,{ }^{*} P<0.01$

partitioned into nuclear and cytoplasmic fractions, we found that HAGLROS was localized preferentially to the cytoplasm (Fig. 5a). FISH experiments also showed that HAGLROS was mostly located in the cytoplasm (Fig. 5b) and further confirmed that HAGLROS functioned as a mainly cytoplasmic lncRNA. It is well known that cytoplasmic lncRNAs can bind directly to miRNA and function as sponges or compete with ceRNAs to control the availability of miRNA for binding to their target mRNAs [26, 27]. Bioinformatics analysis using miRcode (http:// www.mircode.org/) and StarBase v2.0 (http://starbase.sysu.edu.cn/mirLncRNA.php) software suggested that HAGLROS could bind both miR-100-5p and the Argonaute 2 (Ago2) protein. Based on this prediction, we speculated that HAGLROS might act as a sponge in GC. In the GC cells, HAGLROS knockdown by siRNAs caused miR-100-5p up-regulation (Fig. 5c), while overexpression of miR-100-5p by transfection with mimics caused HAGLROS down-regulation (Fig. 5d). These results show that HAGLROS and miR-100-5p are competitively expressed. Moreover, the expression of miR-100-5p and HAGLROS showed an inverse correlation in tumor samples in the higher expression of HAGLROS in GC compared to the adjacent non-cancerous tissue (Fig. 5e).

To further determine the interaction of miR-100-5p and HAGLROS, we constructed luciferase vectors of wild-type and mutant HAGLROS (the binding site for miR-100-5p was mutated). Using a dual-luciferase 


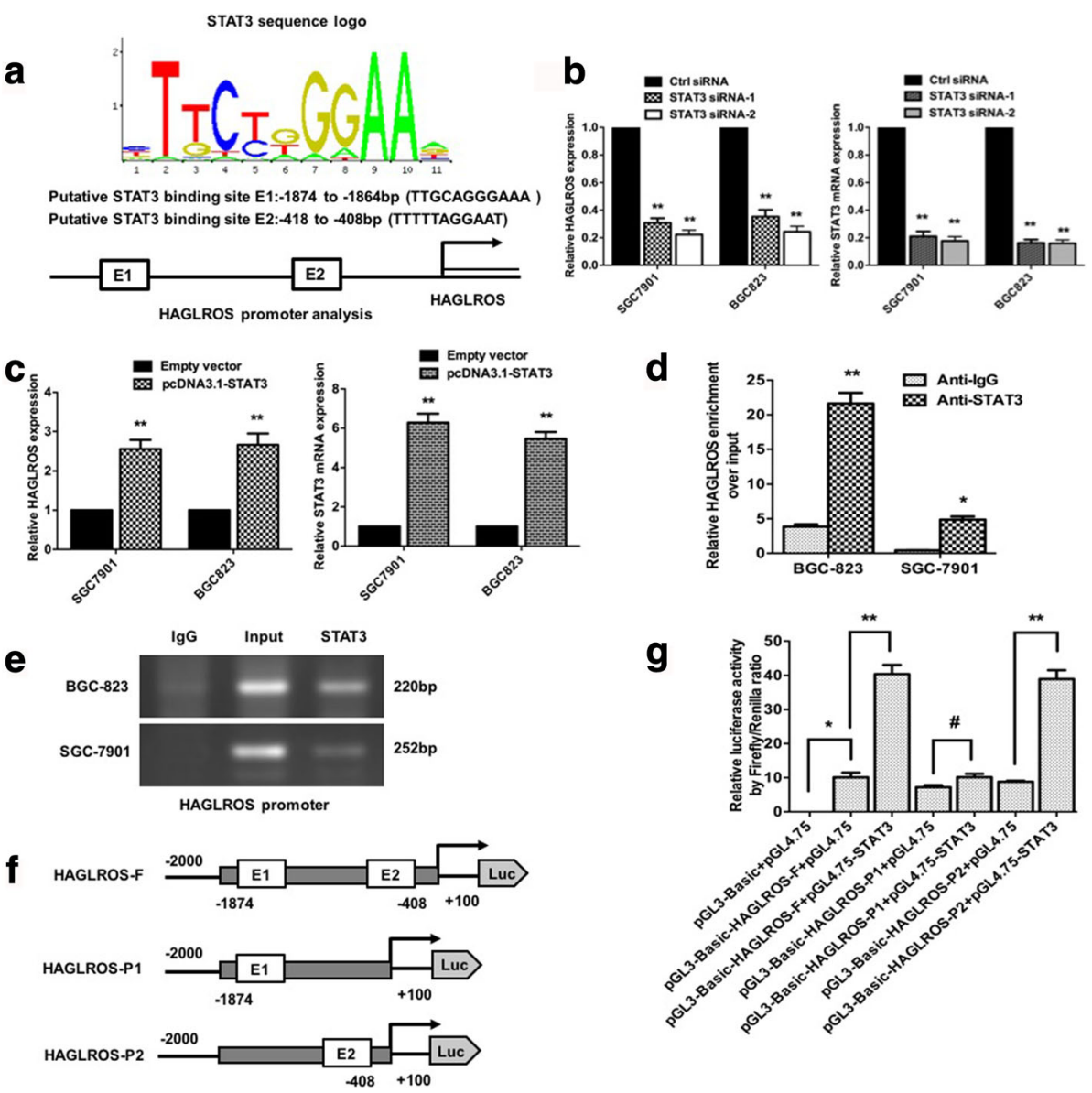

Fig. 4 STAT3 induces HAGLROS expression as a transcription factor. a The predicted sites of STAT3-binding in human HAGLROS promoter by gene sequence analysis. $\mathbf{b}$ The effect of STAT3 knockdown on HAGLROS expression and the transfection efficiency of STAT3 siRNAs. $\mathbf{c}$ The effect of STAT3 overexpression on HAGLROS expression and the transfection efficiency of STAT3 plasmid. $\mathbf{d}$ Quantitative ChIP assays were used to show direct binding of STAT3 to endogenous HAGLROS promoter regions. e The representative blot of the binding of STAT3 to endogenous HAGLROS promoter regions. $\mathbf{f}$ and $\mathbf{g}$ A dual-luciferase reporter assay was performed by co-transfecting the full-length HAGLROS promoter (HAGLROS-F) or deleted HAGLROS E1 or E2 fragment (HAGLROS-P2 and HAGLROS-P1) with STAT3 plasmid or empty vectors in 293T cells. Error bars indicate the means \pm S.E.M. ${ }^{*} P<0.05,{ }^{* *} P<0.01, \# P>0.05$

reporter assay, we found that $293 \mathrm{~T}$ cells transfected with wild-type HAGLROS and miR-100-5p mimics, but not the mutant HAGLROS, presented significantly decreased luciferase activity (Fig. 5f). We performed anti-AGO2 RIP to detect whether HAGLROS was regulated by miR-100-5p in an AGO2-dependent manner. Endogenous HAGLROS pull-down by AGO2 was significantly enriched in miR-100-5p-overexpressing cells (Fig. 5g).

\section{HAGLROS functions as a ceRNA to antagonize miR-100- 5p-mediated mTOR mRNA degradation}

To identify potential functional targets of HAGLROS, we performed transcript RNA high-throughput sequencing. As shown in Additional file 6: Figure S3a, many genes involved in cell proliferation and autophagy were affected by HAGLROS. We confirmed expressions of a panel of genes which were up- and down-regulated by HAGLROS knockdown using qRT-PCR assays (Additional file 7: Figure S4). Furthermore, GO-term and pathway enrichment analysis showed that the differentially expressed genes about autophagy indeed enriched at a significant level by HAGLROS silencing (Additional file 6: Figure S3b and c). Of all the identified genes, mTOR and ATG9A/9B (Fig. 6a) are known to be autophagy-related signals. Bioinformatics analysis using miRcode (http://www.mircode.org/) and StarBase v2.0 (http://starbase.sysu.edu.cn/ mirLncRNA.php) software suggested that HAGLROS could bind both miR-100-5p and mTOR mRNA (Fig. 6b). The fact that transfection of miR-100-5p mimics attenuated mTOR mRNA levels (Fig. 6c) suggested that mTOR might be the target of miR-100-5p. To verifying this hypothesis, the 3 '-UTR of mTOR was cloned into a luciferase vector and transfected into 293T cells together with 

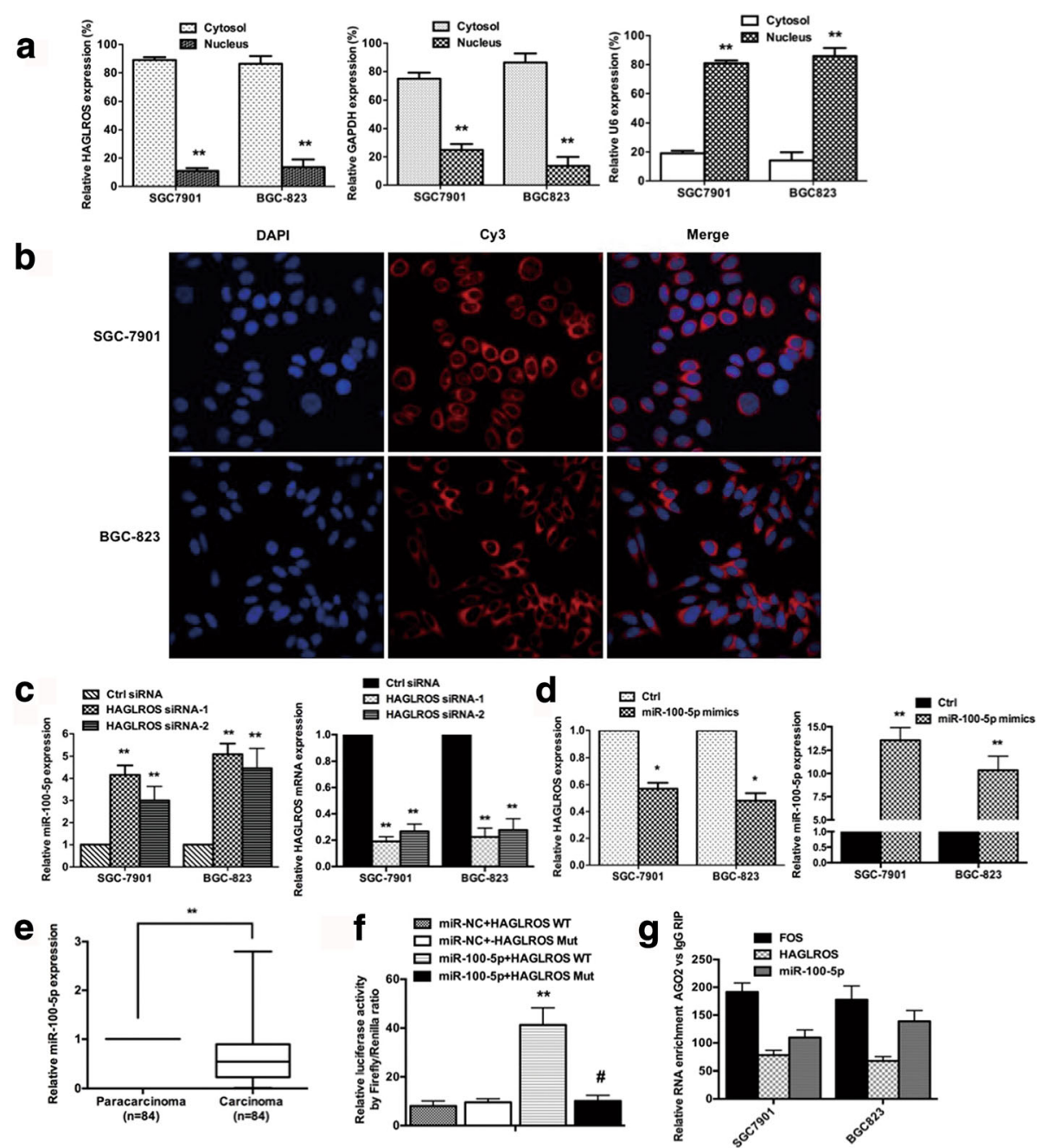

Fig. 5 Subcellular localization of HAGLROS and its "sponge" function as a ceRNA competing with miR-100-5p. a RNA was extracted from the nuclear and the cytoplasmic fractions of SGC-7901 and BGC-823 cells and HAGLROS expression of the nuclear and the cytoplasmic fraction was measured by qRT-PCR. GAPDH was used as a cytosolic marker, and U6 was used as a nuclear marker. b FISH was used to confirm HAGLROS location in SGC-7901 and BGC-823 cells, using Cy3 probes for HAGLROS, DAPI for nuclear staining. c miR-100-5p expression was examined in SGC-7901 and BGC-823 cells with HAGLROS knockdown by siRNAs, and HAGLROS expression was tested to determine the transfection efficiencies. d HAGLROS levels were examined in SGC-7901 and BGC-823 cells transfected with miR-100-5p, and miR-100-5p levels were tested for transfection efficiencies. e The expression of miR-100-5p in tumor samples of GC compared to adjacent non-cancerous tissues. $\mathbf{f}$ Wild-type or mutant HAGLROS plasmid was co-transfected with miR-NC or miR-100-5p mimics into 293T cells, and relative luciferase activities were measured to determine the level of interaction between miR-100-5p and HAGLROS. g RNA levels in immunoprecipitates are presented as fold enrichment relative to $\operatorname{lgG}_{\mathrm{g}}$ in $\mathrm{AGO}_{2}$ cells by RIP experiment. Error bars indicate the means \pm S.E.M. ${ }^{*} P<0.05,{ }^{* *} P<0.01, \# P<0.05$

miR-100-5p mimics, an miR-100-5p inhibitor or a negative control. The miR-100-5p mimics significantly reduced the luciferase activity, while the miR-100-5p inhibitor markedly strengthened it, indicating that mTOR was a direct target of miR-100-5p. Subsequently, the 3'-UTR of mTOR was co-transfected with the HAGLROS plasmid and miR-100-5p mimics. HAGLROS overexpression negated the decrease in luciferase activity induced by overexpressing miR-100-5p (Fig. 6d). This result implied that HAGLROS bound to miR-100-5p and released mTOR from miR-100-5p, further demonstrating the existence of
HAGLROS-mTOR crosstalk through competition for miR-100-5p binding.

\section{HAGLROS interacted with mTORC1 through binding with components of the complex}

To verify crosstalk of the cytoplasmic IncRNA HAGLROS and mTOR, we performed bioinformatics analysis using a web of RNA and protein interactions (http://pridb.gdcb.iastate.edu/RPISeq/) and found that the mTORC1 components mTOR, Raptor and PRAS40 could possibly interact with HAGLROS. In our analysis, both the RF 

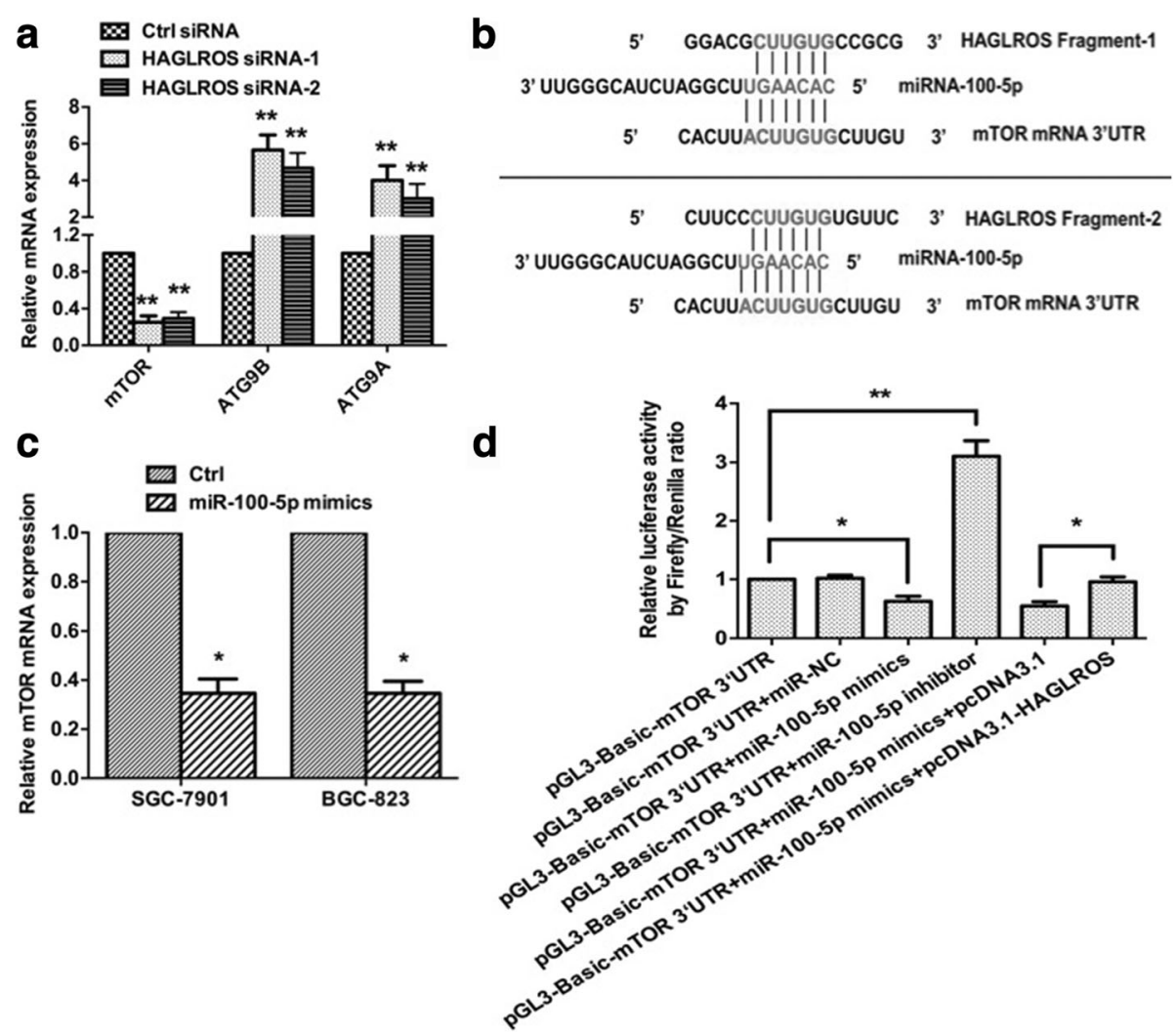

Fig. 6 HAGLROS antagonized miR-100-5p-mediated mTOR mRNA degradation. a The relative expression levels of autophagy-related signals mTOR and ATG9A/9B were validated by qRT-PCR upon HAGLROS knockdown in accordance with RNA high-throughput sequencing guidelines. $\mathbf{b}$ Bioinformatic analysis of the interactions of HAGLROS with miR-100-5p and mTOR mRNA. c The effect of miR-100-5p overexpression by transfection with miR-100-5p mimics on mTOR mRNA level in GC cells. d Relative luciferase activity of mTOR mRNA 3'-UTR was determined after transfection with miR-100-5p mimics, miR-100-5p inhibitor or HAGLROS plasmid. Error bars indicate the means \pm S.E.M. ${ }^{*} P<0.05,{ }^{* *} P<0.01$

classifier and the SVM classifier were larger than 0.5 (Fig. 7a). RIP experiments examined and confirmed the interaction of HAGLROS directly with mTOR, Raptor and PRAS40 in GC cells (Fig. 7b). Moreover, an RNA pulldown assay further identified that HAGLROS indeed bound with mTOR, Raptor and PRAS40 in GC cells (Fig. 7c and d).

\section{HAGLROS promotes GC progression through mTOR- mediated autophagy inhibition}

Usually, mTOR mediates signaling from its effectors, maintaining normal cell function and homeostasis. However, in various diseases, especially in cancer, this capacity is lost because of mutations or activation of signals upstream of mTOR that lead to persistent proliferation and tumor growth [28]. It is well known that mTORC1 inhibits autophagy through its interaction with autophagyrelated genes. Western blot analysis showed that knockdown of HAGLROS markedly down-regulated mTORC1 activity and alleviated the phosphorylation of both mTOR and downstream molecules in BGC-
823 cell lines (Fig. 8a). Upon HAGLROS knockdown, the autophagy markers LC3 and P62 underwent respective changes: the LC3-I to LC3-II transition increased, and P62 levels decreased (Fig. 8b). Immunofluorescence assay demonstrated that LC3-II punctuation was markedly elevated in BGC-823 cells transfected with HAGLROS siRNAs compared to Ctrl siRNA (Fig. 8c). Furthermore, we found increasing mTOR pathway members in HAGLROS silenced cells inhibited the autophagic phenotype (Additional file 8: Figure S5a). All these findings suggested that HAGLROS could inhibit autophagy through activating mTORC1 signals, at least in part. Therefore, we presumed that HAGLROS contributed to GC development by inhibiting autophagy, and the next investigation confirmed this hypothesis. We treated BGC-823 cells with the autophagic inhibitor 3-methyladenine (3-MA) and found that cell viability and migratory ability were obviously increased (Fig. 8d, e and Additional file 8: Figure S5b). These results validate that in GC cells, inhibition of autophagy promotes tumor development, at least in part. Taken together, these results indicate that HAGLROS contributes 

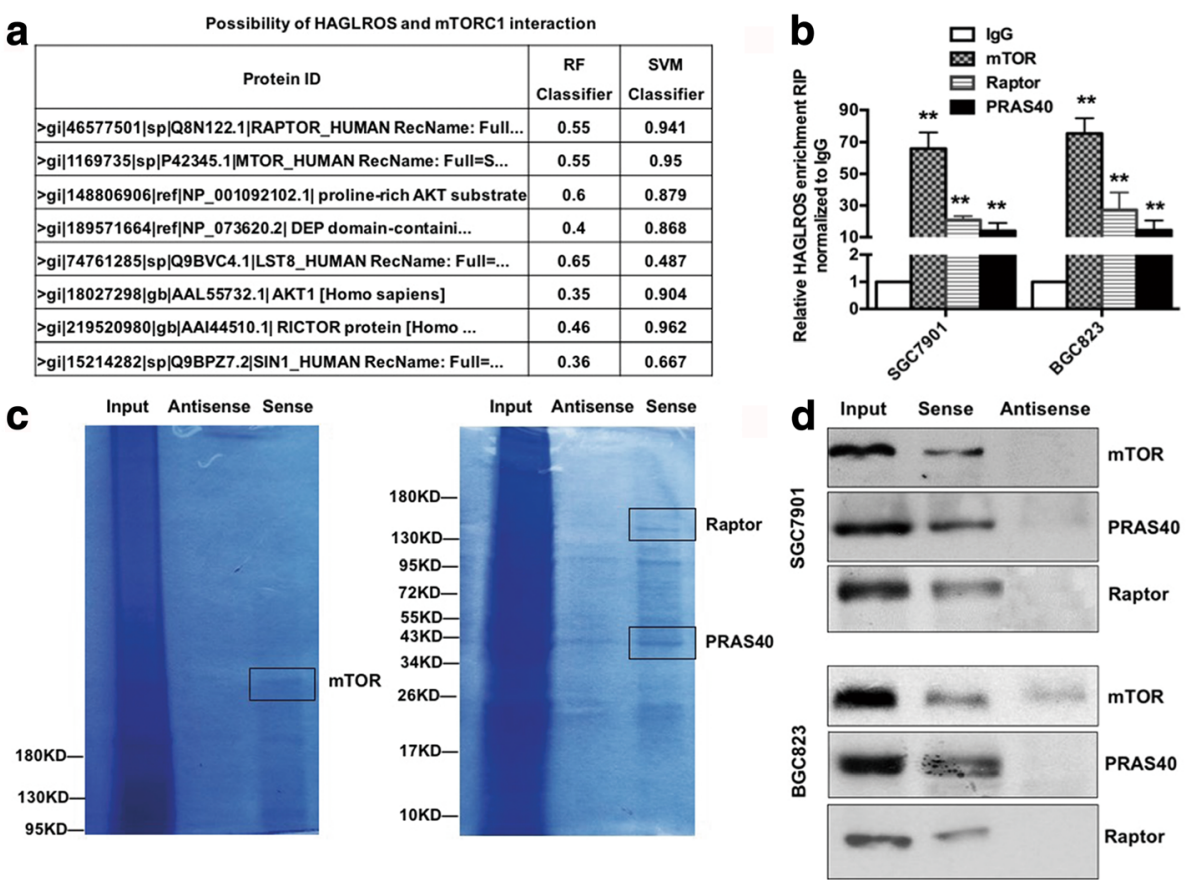

Fig. 7 Bioinformatics prediction and experimental verification of HAGLROS binding to mTORC1 components. a Bioinformatic analysis of HAGLROS interacting with mTORC1 components. Values greater than 0.5 for both the RF classifier and the SVM classifier indicate a high possibility of interaction. b HAGLROS levels in immunoprecipitates are presented as fold enrichment in mTOR, Raptor and PRAS40 antibodies relative to lgG immunoprecipitates by RIP experiments. c Coomassie brilliant blue staining of mTOR, Raptor and PRAS40 protein levels by SDS-PAGE of immunoprecipitates from HAGLROS pulldown. d Western blot analysis of mTOR, Raptor and PRAS40 protein levels in immunoprecipitates from HAGLROS pulldown. ${ }^{* *} P<0.01$

to GC proliferation and migration by mTORC1-mediated autophagy inhibition.

\section{Discussion}

Most cancers are genetic diseases that change the flow of cellular information and thus disrupt cellular homeostasis and promote proliferation. More and more evidence confirms that lncRNAs have important roles in GC carcinogenesis, proliferation and metastasis through survival signaling pathways [29]. Differential display analysis of human prostate cancers identified Differential display 3, also known as PCA3, this lncRNA was approved by the Food and Drug Administration (FDA) for prostate cancer diagnosis and was the first case of a IncRNA being used for clinical testing by FDA approval [30, 31]. Similarly, analyses of gastric secretions from patients with GC identified IncRNA-AA174084 as a biomarker capable of differentiating between GC and benign disorders of the gastric epithelium [32]. Many lncRNAs have been reported to be associated with GC, but the most characteristic biomarker remains unclear. In the present study, we found that the lncRNA HAGLROS was significantly overexpressed in GC compared to corresponding non-tumor tissues. The high expression levels of HAGLROS in GC were positively correlated with invasion depth and TNM stage. In addition, high
HAGLROS expression in GC tissues was associated with a poor prognosis and could be an independent prognostic indicator. These results suggested that HAGLROS might have important roles in GC progression.

The dysregulation of IncRNAs influences various pathological processes. Alike protein-coding transcripts, the transcription of IncRNAs is subject to typical epigenetic-mediated and transcription factor-mediated regulation. STAT3 is both a transcriptional activator and an oncogene under normal physiological conditions. However, much evidence indicates that STAT3 is constitutively activated in cancers, playing a crucial role in tumor onset and progression. In addition to its traditional role in cancer cell proliferation, invasion, and migration, STAT3, as a transcription factor, promotes cancer development by altering the expression of other genes in cancer cells [33]. Furthermore, overexpression of STAT3 has been observed in various types of tumors, including GC. In this study, we found that HAGLROS was a direct target of transcription factor STAT3, which was affirmed by STAT3 binding to the predicted site of the promoter region of HAGLROS and by STAT3 causing significant induction of HAGLROS promoter activity, as determined by luciferase reporter assay (Fig. 4). Therefore, up-regulation of HAGLROS in GC is partly due to STAT3 activation during tumor progression. 

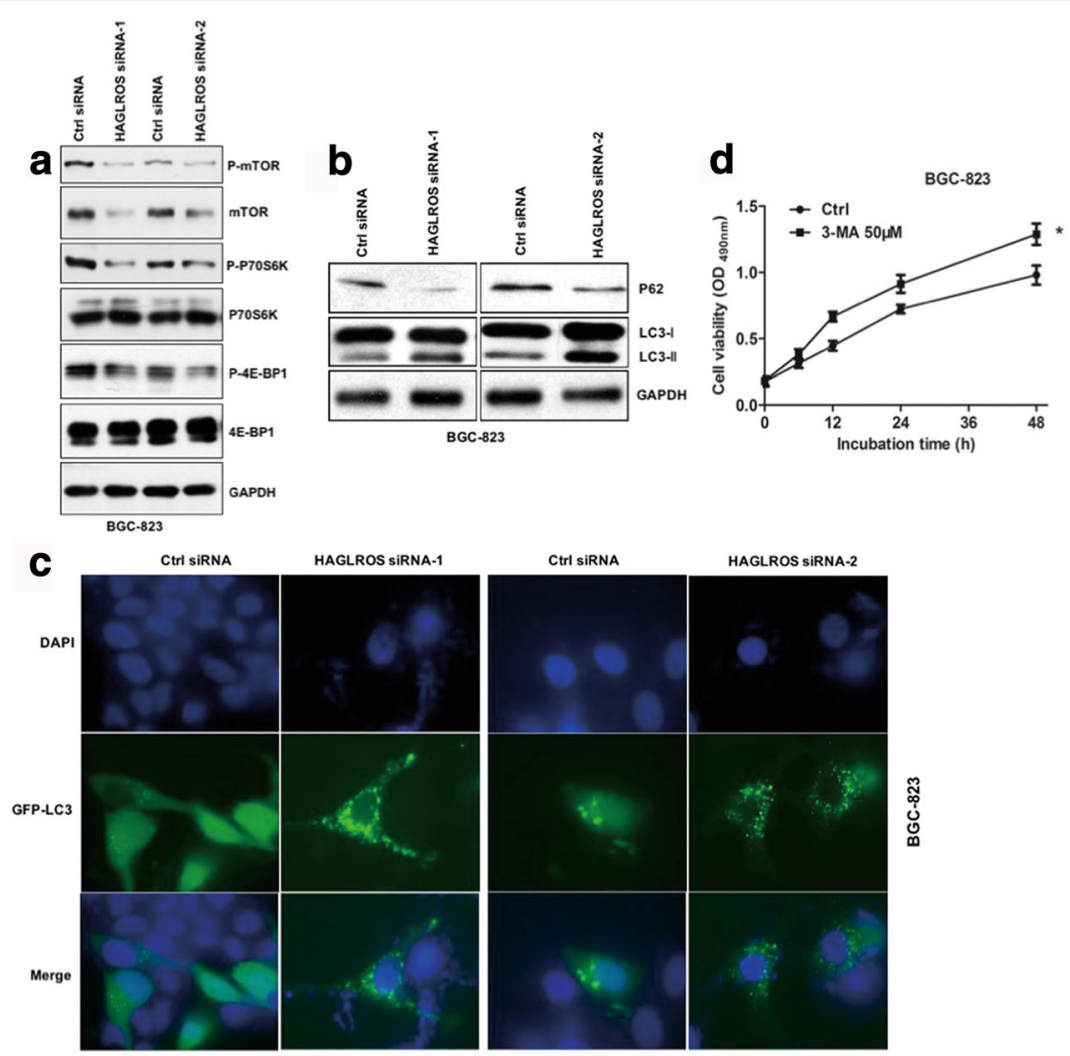

HAGLROS SIRNA-1
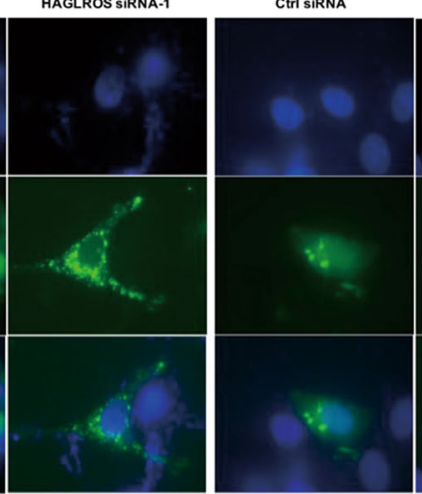

HAGLROS SIRNA-2
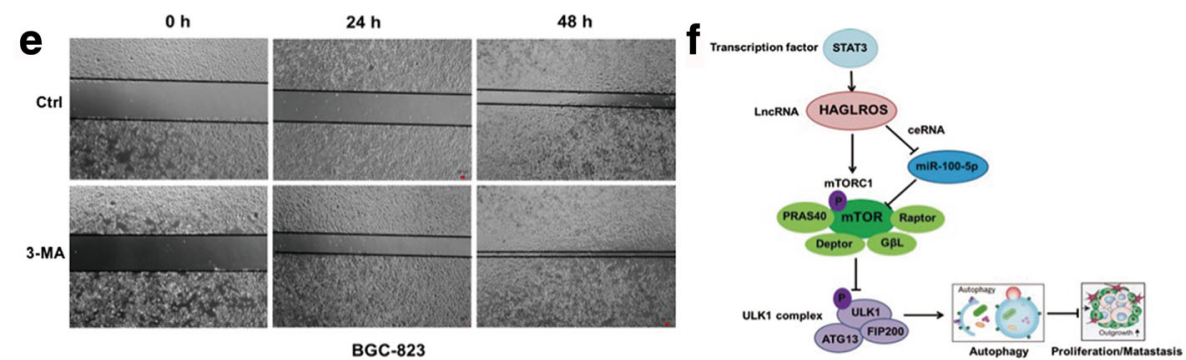

Fig. 8 HAGLROS promotes GC progression through mTOR-mediated autophagy inhibition. a Upon knockdown of HAGLROS by siRNAs, mTORC1 activity, including phosphorylation of both mTOR and downstream molecules, was analyzed in BGC-823 cell lines by Western blot. b Autophagy markers P62 and LC3 were measured by Western blot in BGC-823 cell lines with HAGLROS knockdown. c LC3-II punctuation was determined by BGC-823 cells co-transfected with GFP-LC3 plasmid and HAGLROS siRNAs by immunofluorescence assays. $\mathbf{d}$ and (e) BGC-823 cells were treated with the autophagic inhibitor 3-methyladenine (3-MA), and cell viability and migratory ability were measured by MTT assay (d) and (e) wound scratch assay. $\mathbf{f}$ Summary of the molecular mechanisms of HAGLROS in gastric cancer cells. ${ }^{*} P<0.05$

lncRNA regulation of cellular processes depends in part on IncRNA cellular localization: nuclear IncRNAs are enriched for functionality involving chromatin interactions, transcriptional regulation, and RNA processing, while cytoplasmic lncRNAs can modulate mRNA stability or translation and influence cellular signaling cascades [1]. LncRNA modulation of RNA metabolism is an emerging theme for lncRNAs that are enriched in the cytoplasm, where the IncRNAs participate in cellular biological processes by functioning as ceRNAs or "RNA sponges" regulating mRNA stability, mRNA alternative splicing, and protein localization [34]. In the present study, we report that HAGLROS is localized preferentially in the cytoplasm, as determined by subcellular fractionation and FISH experiments. HAGLROS functions as a ceRNA to antagonize miR-100-5p-mediated mTOR mRNA degradation; HAGLROS and mTOR interact through competition for miR-100-5p binding. In addition, we observed that HAGLROS, as a mainly cytoplasmic lncRNA, interacts directly with mTORC1 components (mTOR, Raptor and PRAS40) and activated the mTORC1 pathway by stabilizing the complex' structure. In accordance with the above statements, cytoplasmic lncRNA HAGLROS has two mechanisms to activate the 
mTOR pathway and thus inhibit autophagy. On the one hand, HAGLROS functions as a ceRNA to increase mTOR mRNA expression through competing with miR-100-5p, on the other hand, HAGLROS binds mTORC1 key proteins to activate the complex and finally participates in cellular biological processes. mTORC1, a master positive regulator of cell growth and proliferation, forms the integrational hub of an extensive network of regulatory proteins that transmit extrinsic and intrinsic signals regarding cellular nutritional status. The influence of mTORC1 on cellular metabolism is substantial considering its regulation by common oncogenic signaling pathways (e.g., PIK3CAAKT1 and RAS-ERK) [35] and the observation that aberrant mTORC1 signaling is found in $40 \%$ to $90 \%$ of human cancers [36].

Given the intimate relationship between mTORC1 signaling and autophagy, it is likely that cancer-associated sequence changes in the mechanistic target of rapamycin or mTOR and/or aberrant mTOR protein expression would perturb autophagy, making autophagy an important mediator of the effects of this common dysregulation in human cancer [37]. Here, we monitored the knockdown of HAGLROS and its effects on autophagy through the mTOR pathway. The functional relevance of autophagy in tumor formation and progression remains controversial. Intriguingly, many oncogenes and tumor suppressor genes affect autophagic pathways, and the dysregulation of the autophagic process contributes to malignant transformation [38]. Many tumor suppressor proteins, such as p53, phosphatase and tensin homo$\log$ (PTEN) and death-associated protein kinase (DAPK), that provide constitutive input signals to activate autophagy are mutated in multiple cancers. Conversely, oncogenes, including Akt, mTOR and Bcl-2, inhibit autophagic processes indicating that elevated autophagy signaling may contribute to tumor suppression $[39,40]$. In GC cells, HAGLROS and mTOR levels are consistently parallel, with increased levels inhibiting autophagy and promoting tumor proliferation and invasion. The regulatory mechanism of HAGLROS was summarized in the Fig. 8f.

\section{Conclusions}

Our study demonstrates that the GC-associated lncRNA HAGLROS is an oncogenic lncRNA that promotes tumorigenesis and progression through mTOR pathwaymediated autophagy suppression by serving as a ceRNA for miR-100-5p and as a cytoplasmic scaffold to bind mTORC1. Our findings support the idea that lncRNAs such as HAGLROS play crucial roles in GC progression and prove that HAGLROS is a potential effective target for treating GC.

\section{Additional files}

Additional file 1: Table S1. The relationship between HAGLROS expression and clinicopathological factors of GC patients. (DOCX $14 \mathrm{~kb}$ )

Additional file 2: Table S2. Primers used for $q R T-P C R, R T-P C R, C H I P$ and siRNAs/shRNA oligonucleotides. (XLSX $12 \mathrm{~kb}$ )

Additional file 3: Figure S1. The relative IncRNAs expression from 12 GC patients were validated by qRT-PCR. Error bars indicate the means \pm S.E.M. ${ }^{*} P<0.05$, ${ }^{* *} P<0.01$ for carcinoma vs paracarcinoma. (TIFF $165 \mathrm{~kb}$ )

Additional file 4: Table S3. Univariate and multivariate analyses of the clinicopathological factors for overall survival in 84 patients with GC. (DOCX 16 kb)

Additional file 5: Figure S2. (a) Relative areas of the wound scratch assay by Image J software. ${ }^{*} P<0.05$ for siRNAs vs Ctrl siRNAs and pCDNA3.1-HAGLROS vs vector. (b) Transcription efficiencies of siRNAs, shRNAs and pcDNA3.1-HAGLROS. ${ }^{* *} P<0.01$. Error bars indicate the means \pm S.E.M. (TIFF $1170 \mathrm{~kb}$ )

Additional file 6: Figure S3. RNA-sequencing analysis of HAGLROS siRNA vs Ctrl. (a) 194 differentially expressed genes upon HAGLROS siRNA vs Ctrl. (b) GO analysis of differentially expressed genes. (c) Pathway enrichment analysis of differentially expressed genes. (TIFF $3460 \mathrm{~kb}$ )

Additional file 7: Figure S4. The relative expression levels of downstream signals were validated by qRT-PCR upon HAGLROS knockdown in accordance with RNA high-throughput sequencing guidelines. (a) Down-regulated genes were validated by qRT-PCR upon HAGLROS knockdown. (b) Up-regulated genes were validated by qRT-PCR upon HAGLROS knockdown. Error bars indicate the means \pm S.E.M. ${ }^{*} P<0.05,{ }^{* *} P<0.01$. (TIFF $1840 \mathrm{~kb}$ )

Additional file 8: Figure S5. (a) Increasing mTOR pathway members in HAGLROS silenced cells inhibited the autophagic phenotype. (b) Relative areas of the wound scratch assay by Image J software, corresponding Fig. 8e. (TIFF $1880 \mathrm{~kb}$ )

\section{Abbreviations}

3-MA: 3-methyladenine; Ago2: Argonaute 2; ceRNA: Competing endogenous RNA; ChIP: Chromatin immunoprecipitation; DAPK: Death-associated protein kinase; DEPTOR: DEP domain containing mTOR-interacting protein; FDA: Food and Drug Administration; GC: Gastric cancer; GEO: Gene Expression Omnibus; IHC: Immunohistochemical; IncRNAs: Long noncoding RNAs; mLST8: Mammalian lethal with sec-13 protein 8; mSin1: Mammalian stress-activated map kinase-interacting protein 1; mTOR: Mammalian target of rapamycin; mTORC1: mTOR complex 1; mTORC2: mTOR complex 1; NCBI: National Center for Biotechnology Information; ncRNAs: Noncoding RNAs; PRAS40: Proline-rich Akt substrate 40 kDa; protor1/2: Protein observed with rictor 1 and 2; PTEN: Phosphatase and tensin homolog; Raptor: Regulatory-associated protein of mammalian target of rapamycin; Rictor: Rapamycin-insensitive companion of mTOR; RIP: RNA immunoprecipitation; TCGA: The Cancer Genome Atlas

\section{Acknowledgements}

Not applicable

\section{Funding}

This work was supported by grants from the National Natural Science Foundation of China (no. 31401094, no. 81572928 and no. 81772978) and the National Key Research and Development Program of China (2017YFC1308900).

\section{Availability of data and materials}

The datasets used and/or analyzed during the current study are available from the corresponding author on reasonable request.

\section{Authors' contributions}

Conception and design: FY and WD. Development of the methodology: RX. Acquisition of data: FY, JFC and PW. Analysis and interpretation of data: JY, and RX. Writing and revision of the manuscript: FY. Administrative, technical, and material support: XYH, DYG and CJT. All authors read and approved the final manuscript. 


\section{Ethics approval and consent to participate}

The research protocol was reviewed and approved by the Human Research Ethics Committee of Nanjing Medical University and written informed consent was obtained from each patient included in the study.

\section{Consent for publication}

Not applicable

\section{Competing interests}

The authors declare that they have no competing interests.

\section{Publisher's Note}

Springer Nature remains neutral with regard to jurisdictional claims in published maps and institutional affiliations.

\section{Author details}

'Department of Oncology, Nanjing First Hospital, Nanjing Medical University, Nanjing, People's Republic of China. ${ }^{2}$ Department of Clinical Laboratory, Nanjing Chest Hospital, Nanjing, People's Republic of China. ${ }^{3}$ Department of Biochemistry and Molecular Biology, School of Basic Medical Sciences, Nanjing Medical University, Nanjing 211166, People's Republic of China.

\section{Received: 18 September 2017 Accepted: 28 December 2017} Published online: 12 January 2018

\section{References}

1. Batista PJ, Chang HY. Long noncoding RNAs:cellular address codes in development and disease. Cell. 2013;152(6):1298-307.

2. Djebali S, Davis CA, Merkel A, Dobin A, Lassmann T, Mortazavi A, Tanzer A, Lagarde J, Lin W, Schlesinger F, et al. Landscape of transcription in human cells. Nature. 2012;489(7414):101-8.

3. Fatica A, Bozzoni I. Long non-coding RNAs: new players in cell differentiation and development. Nat Rev Genet. 2014;15(1):7-21.

4. Colquhoun A, Arnold M, Ferlay J, Goodman KJ, Forman D, Soerjomataram I. Global patterns of cardia and non-cardia gastric cancer incidence in 2012. Gut. 2015;64(12):1881-8.

5. Torre LA, Siegel RL, Ward EM, Jemal A. Global cancer incidence and mortality rates and trends-an update. Cancer Epidemiol Biomark Prev. 2016; 25(1):16-27.

6. Siegel RL, Miller KD, Jemal A. Cancer statistics, 2017. CA Cancer J Clin. 2017; 67(1):7-30.

7. Liu S, Song L, Zeng S, Zhang L. MALAT1-miR-124-RBG2 axis is involved in growth and invasion of HR-HPV-positive cervical cancer cells. Tumor Biol. 2016:37(1):633-40.

8. Lu Z, Xiao Z, Liu F, Cui M, Li W, Yang Z, Li J, Ye L, Zhang X. Long non-coding RNA HULC promotes tumor angiogenesis in liver cancer by up-regulating sphingosine kinase 1 (SPHK1). Oncotarget. 2016;7(1): 241-54.

9. Wu M, Lin Z, Li X, Xin X, An J, Zheng Q, Yang Y, HULC LD. Cooperates with MALAT1 to aggravate liver cancer stem cells growth through telomere repeat-binding factor 2. Sci Rep. 2016;6:36045.

10. Yuan JH, Yang F, Wang F, Ma JZ, Guo YJ, Tao QF, Liu F, Pan W, Wang TI, Zhou CC, et al. A long noncoding RNA activated by TGF-beta promotes the invasion-metastasis cascade in hepatocellular carcinoma. Cancer Cell. 2014; 25(5):666-81.

11. Quagliata L, Matter MS, Piscuoglio S, Arabi L, Ruiz C, Procino A, Kovac M, Moretti F, Makowska Z, Boldanova T, et al. Long noncoding RNA HOTTIP/HOXA13 expression is associated with disease progression and predicts outcome in hepatocellular carcinoma patients. Hepatology. 2014;59(3):911-23.

12. Gupta RA, Shah N, Wang KC, Kim J, Horlings HM, Wong DJ, Tsai MC, Hung $T$, Argani P, Rinn JL, et al. Long non-coding RNA HOTAIR reprograms chromatin state to promote cancer metastasis. Nature. 2010;464(7291):1071-6.

13. Shi $X$, Sun M, Liu H, Yao Y, Kong R, Chen F, Song YA. Critical role for the long non-coding RNA GAS5 in proliferation and apoptosis in non-small-cell lung cancer. Mol Carcinog. 2015;54(Suppl 1):E1-E12.

14. Yu G, Yao W, Gumireddy K, Li A, Wang J, Xiao W, Chen K, Xiao H, Li H, Tang $\mathrm{K}$, et al. Pseudogene PTENP1 functions as a competing endogenous RNA to suppress clear-cell renal cell carcinoma progression. Mol Cancer Ther. 2014; 13(12):3086-97.
15. Lennox KA, Behlke MA. Cellular localization of long non-coding RNAs affects silencing by RNAi more than by antisense oligonucleotides. Nucleic Acids Res. 2016;44(2):863-77.

16. Mas-Ponte D, Carlevaro-Fita J, Palumbo E, Hermoso Pulido T, Guigo R, Johnson R. LncATLAS database for subcellular localization of long noncoding RNAs. RNA. 2017:23(7):1080-7.

17. Cesana M, Cacchiarelli D, Legnini I, Santini T, Sthandier O, Chinappi M, Tramontano A, Bozzoni IA. Long noncoding RNA controls muscle differentiation by functioning as a competing endogenous RNA. Cell. 2011; 147(2):358-69.

18. Poliseno L, Salmena L, Zhang J, Carver B, Haveman WJ, Pandolfi PPA. Coding-independent function of gene and pseudogene mRNAs regulates tumour biology. Nature. 2010;465(7301):1033-8.

19. Zhang F, Zhang L, Zhang C. Long noncoding RNAs and tumorigenesis: genetic associations, molecular mechanisms, and therapeutic strategies. Tumour Biol. 2016;37(1):163-75.

20. Wang KC, Chang HY. Molecular mechanisms of long noncoding RNAs. Mol Cell. 2011;43(6):904-14.

21. Laplante M, Sabatini DM. mTOR signaling in growth control and disease. Cell. 2012;149(2):274-93.

22. Stephen A. Bustin. Why the need for qPCR publication quidelines? - the case for MIQE. Methods. 2010;50:217-6.

23. Sun M, Nie F, Wang Y, Zhang Z, Hou J, He D, Xie M, Xu L, De W, Wang Z, et al. LnCRNA HOXA11-AS promotes proliferation and invasion of gastric cancer by scaffolding the chromatin modification factors PRC2, LSD1, and DNMT1. Cancer Res. 2016;76(21):6299-310.

24. Yang F, Deng R, Qian XJ, Chang SH, XQ W, Qin J, Feng GK, Ding K, Zhu XF. Feedback loops blockade potentiates apoptosis induction and antitumor activity of a novel AKT inhibitor DC120 in human liver cancer. Cell Death Dis. 2014:5:e1114.

25. Yang F, Tang XY, Liu H, Jiang ZW. Inhibition of mitogen-activated protein kinase signaling pathway sensitizes breast cancer cells to endoplasmic reticulum stress-induced apoptosis. Oncol Rep. 2016;35(4): $2113-20$.

26. Zhou X, Gao Q, Wang J, Zhang X, Liu K, Duan Z. Linc-RNA-RoR acts as a "sponge" against mediation of the differentiation of endometrial cancer stem cells by microRNA-145. Gynecol Oncol. 2014;133(2):333-9.

27. Salmena L, Poliseno L, Tay Y, Kats L, Pandolfi PPA. ceRNA hypothesis: the Rosetta stone of a hidden RNA language? Cell. 2011;146(3):353-8.

28. Willems L, Tamburini J, Chapuis N, Lacombe C, Mayeux P, Bouscary D. PI3K and mTOR signaling pathways in cancer: new data on targeted therapies. Curr Oncol Rep 2012; 14(2):129-138.

29. Okugawa Y, Toiyama Y, Hur K, Toden S, Saigusa S, Tanaka K, Inoue Y, Mohri $\mathrm{Y}$, Kusunoki M, Boland CR, et al. Metastasis-associated long non-coding RNA drives gastric cancer development and promotes peritoneal metastasis. Carcinogenesis. 2014;35(12):2731-9.

30. Bussemakers MJ, van Bokhoven A, Verhaegh GW, Smit FP, Karthaus HF, Schalken JA, Debruyne FM, Ru N, Isaacs WB. DD3: a new prostate-specific gene, highly overexpressed in prostate cancer. Cancer Res. 1999;59(23): 5975-9.

31. Wei JT, Feng Z, Partin AW, Brown E, Thompson I, Sokoll L, Chan DW, Lotan Y, Kibel AS, Busby JE, et al. Can urinary PCA3 supplement PSA in the early detection of prostate cancer? J Clin Oncol. 2014;32(36):4066-72.

32. Shao Y, Ye M, Jiang X, Sun W, Ding X, Liu Z, Ye G, Zhang X, Xiao B, Guo J. Gastric juice long noncoding RNA used as a tumor marker for screening gastric cancer. Cancer. 2014;120(21):3320-8.

33. Yuan J, Zhang F, Niu R. Multiple regulation pathways and pivotal biological functions of STAT3 in cancer. Sci Rep. 2015:5:17663.

34. Schmitt AM, Chang HY. Long noncoding RNAs in cancer pathways. Cancer Cell. 2016;29(4):452-63.

35. Yecies JL, Manning BD. mTOR links oncogenic signaling to tumor cell metabolism. J Mol Med (Berl). 2011;89(3):221-8.

36. Menon S, Manning BD. Common corruption of the mTOR signaling network in human tumors. Oncogene. 2008;27(Suppl 2):S43-51.

37. Wang S, Livingston MJ, Su Y, Dong Z. Reciprocal regulation of cilia and autophagy via the MTOR and proteasome pathways. Autophagy. 2015;11(4): $607-16$.

38. Bhutia SK, Mukhopadhyay S, Sinha N, Das DN, Panda PK, Patra SK, Maiti TK, Mandal M, Dent P. Wang XY, et al. Autophagy: cancer's friend or foe? Adv Cancer Res. 2013;118:61-95. 
39. Huo Y, Cai H, Teplova I, Bowman-Colin C, Chen G, Price S, Barnard N, Ganesan S, Karantza V, White E, et al. Autophagy opposes p53mediated tumor barrier to facilitate tumorigenesis in a model of PALB2-associated hereditary breast cancer. Cancer Discov. 2013;3(8): 894-907.

40. White E. The role for autophagy in cancer. J Clin Invest. 2015;125(1):42-6.

Submit your next manuscript to BioMed Central and we will help you at every step:

- We accept pre-submission inquiries

- Our selector tool helps you to find the most relevant journal

- We provide round the clock customer support

- Convenient online submission

- Thorough peer review

- Inclusion in PubMed and all major indexing services

- Maximum visibility for your research

Submit your manuscript at www.biomedcentral.com/submit
Biomed Central 